\title{
Artificial Intelligence-Powered Smartphone App to Facilitate Medication Adherence: Protocol for a Human Factors Design Study
}

Don Roosan ${ }^{1 *}$, PharmD, PhD; Jay Chok ${ }^{2}, \mathrm{PhD}$; Mazharul Karim ${ }^{1}$, BPharm; Anandi V Law ${ }^{1}$, BPharm, MS, PhD; Andrius Baskys ${ }^{3}$, MD, PhD; Angela Hwang ${ }^{1}$, PharmD; Moom R Roosan ${ }^{4 *}$, PharmD, PhD

${ }^{1}$ Department of Pharmacy Practice and Administration, College of Pharmacy, Western University of Health Sciences, Pomona, CA, United States

${ }^{2}$ School of Applied Life Sciences, Keck Graduate Institute, Claremont Colleges, Claremeont, CA, United States

${ }^{3}$ Graduate College of Biomedical Sciences, Western University of Health Sciences, Pomona, CA, United States

${ }^{4}$ Department of Pharmacy Practice, School of Pharmacy, Chapman University, Irvine, CA, United States

*these authors contributed equally

\section{Corresponding Author:}

Don Roosan, PharmD, PhD

Department of Pharmacy Practice and Administration

College of Pharmacy

Western University of Health Sciences

309 E 2nd St

Pomona, CA, 91766

United States

Phone: 19094698778

Email:droosan@westernu.edu

\section{Abstract}

Background: Medication Guides consisting of crucial interactions and side effects are extensive and complex. Due to the exhaustive information, patients do not retain the necessary medication information, which can result in hospitalizations and medication nonadherence. A gap exists in understanding patients' cognition of managing complex medication information. However, advancements in technology and artificial intelligence (AI) allow us to understand patient cognitive processes to design an app to better provide important medication information to patients.

Objective: Our objective is to improve the design of an innovative AI- and human factor-based interface that supports patients' medication information comprehension that could potentially improve medication adherence.

Methods: This study has three aims. Aim 1 has three phases: (1) an observational study to understand patient perception of fear and biases regarding medication information, (2) an eye-tracking study to understand the attention locus for medication information, and (3) a psychological refractory period (PRP) paradigm study to understand functionalities. Observational data will be collected, such as audio and video recordings, gaze mapping, and time from PRP. A total of 50 patients, aged 18-65 years, who started at least one new medication, for which we developed visualization information, and who have a cognitive status of 34 during cognitive screening using the TICS-M test and health literacy level will be included in this aim of the study. In Aim 2, we will iteratively design and evaluate an AI-powered medication information visualization interface as a smartphone app with the knowledge gained from each component of Aim 1. The interface will be assessed through two usability surveys. A total of 300 patients, aged 18-65 years, with diabetes, cardiovascular diseases, or mental health disorders, will be recruited for the surveys. Data from the surveys will be analyzed through exploratory factor analysis. In Aim 3, in order to test the prototype, there will be a two-arm study design. This aim will include 900 patients, aged 18-65 years, with internet access, without any cognitive impairment, and with at least two medications. Patients will be sequentially randomized. Three surveys will be used to assess the primary outcome of medication information comprehension and the secondary outcome of medication adherence at 12 weeks.

Results: Preliminary data collection will be conducted in 2021, and results are expected to be published in 2022.

Conclusions: This study will lead the future of AI-based, innovative, digital interface design and aid in improving medication comprehension, which may improve medication adherence. The results from this study will also open up future research 
opportunities in understanding how patients manage complex medication information and will inform the format and design for innovative, AI-powered digital interfaces for Medication Guides.

International Registered Report Identifier (IRRID): PRR1-10.2196/21659

(JMIR Res Protoc 2020;9(11):e21659) doi: 10.2196/21659

\section{KEYWORDS}

artificial intelligence; smartphone app; patient cognition; complex medication information; medication adherence; machine learning; mobile phone

\section{Introduction}

\section{Background}

For decades, the US Food and Drug Administration (FDA) and drug manufacturers provided detailed Medication Guides to the public to effectively communicate crucial patient safety [1]. The guides include warnings, precautions, and other lists of adverse drug reactions [2]. Unfortunately, the information presented in these Medication Guides often contains complicated medical jargon. As a result, the information is difficult to read, especially among patients with lower literacy skills [3]. One study found that only about half of the participants understood the information presented [4]. In another exploratory study, researchers used a mixed methods approach that combines eye-tracking technology, a survey, and qualitative data to explore self-reported measures of drug-risk reading and actual information recall. More than $80 \%$ of participants $(n=29)$ claimed to have read or comprehended only half or more of the risk information. However, eye-tracking measures revealed limited to no understanding of risks and minimal unaided recall [5].

Consequently, patients cannot identify pivotal warnings from the guides, as evidenced by an increase in all medication-related hospitalizations by 117\% from 1997 to 2008 [6]. Limited research in this area suggests that minimal patient engagement with information may contribute to unrecognized adverse effects that can lead to poor adherence. Furthermore, patient cognition and low literacy levels have a negative impact on medication adherence [7-9]. Therefore, the solution may not be whether or not the information was presented but rather how the information presented supports the cognition of the patient [10]. Moreover, understanding the patient's cognitive processes could ultimately impact both intentional and unintentional medication nonadherence. Intentional nonadherence relates to stopping or altering prescribed medications through a perceived purpose, whereas unintentional medication nonadherence relates to specific circumstances that impede compliance, including forgetting to take a dose [11]. Both intentional and unintentional nonadherence lead to poor outcomes and uncontrolled disease states. Additionally, studies have detected a relationship between nonadherence and health literacy $[12,13]$. One study in particular investigated asthmatic patient perspectives of medication nonadherence to inhaled corticosteroids and observed that a factor for noncompliance was suboptimal knowledge of medications [14]. Moreover, health literacy is positively associated with cognition [15]. Thus, patients with lower health literacy and lower cognition have more serious adverse drug events [16]. By understanding cognition, novel solutions to medication nonadherence can potentially be developed. Previous studies in understanding cognitive processes mainly focused on the decision making of clinicians to support cognition [17-19]. Research on the aspects of understanding the patient's cognition is still limited. Thus, a gap exists in the understanding of how patients manage complex medication information cognitively in the digital environment.

Currently, the patient's cognition can be supported through effective medication information communication using digital platforms, such as smartphone apps. In particular, recent advancements in artificial intelligence (AI) embedded into smartphone apps have the potential for a significant impact to monitor and eventually comprehend different features of the patient's cognition [20,21].

We hypothesize that interactive information visualization using human factors design principles delivered through a smartphone platform has the potential to improve medication information retention. Infographics or interactive visualizations using pictures and illustrations have been effective for complex information communication and have proven the age-old saying "a picture is worth a thousand words" [22-25]. Furthermore, AI-based smartphone devices have proven to be effective in clinical trials and chronic disease management to improve medication adherence [21,26]. Utilizing advanced machine learning algorithms, smartphone apps have the potential to improve medication information delivery to patients. Specifically, algorithms based on AI can help advance our understanding of patients' cognitive processes and provide solutions through digital health technology.

\section{Objective}

Our goal is to improve the design of an innovative, AI-powered user interface based on human factors methodology to support the patient's cognition, enhance the ability to comprehend medication information, and ultimately improve medication adherence. The aims are listed below:

1. Aim 1: identify barriers to medication information comprehension.

2. Aim 2: iteratively design and evaluate the AI-powered medication information visualization interface.

3. Aim 3: test the prototype for better comprehension and adherence to medication information. 


\section{Methods}

\section{Overview}

We will use RxNorm and the Unified Medical Language System to design the AI infrastructure to unify medication and biomedical terminologies systematically. Generic medication names for the most prescribed and commonly used oral medications will be obtained from RxNorm. A recursive neural network (RNN)-based methodology was used for developing the AI-based algorithm. Artificial neural networks (ANNs) are modeled after the human brain and structured to recognize and recall information. An RNN is a type of ANN algorithm that operates on structured inputs of an acyclic graph. It is a type of architecture used in machine learning for various applications, such as predicting the weather, processing images, and language composition. We implemented this architecture and used deep learning algorithms using Amazon SageMaker for integrating the AI aspects into the smartphone app. We will use AI to monitor features of user analytics, use predictive tools to understand user interaction, and create a dynamic user environment of the app that changes based on the user's previous input. The deep learning methodology of RNNs will guide us to develop such features. The user's input will be reused to train the machine learning model.

\section{Aim 1: Identify Barriers to Medication Information Comprehension}

\section{Methods Overview}

We will conduct a mixed methods study following these three steps:

1. Step 1: identify patients' perceptions of fear and biases regarding medication information from a focus group study.

2. Step 2: the eye-tracking study will provide design configurations for the interface.

3. Step 3: psychological refractory period (PRP) will help us understand functionalities based on user profile variations.

\section{Step 1: Observational Study}

In the first phase, we will conduct multiple group interviews (ie, focus group methodology) for patients to interact to gain new knowledge and generate meaningful suggestions, opinions, and feedback [27]. Each focus group will have 5 patients. We will ask each patient to provide us with the name of a new medication, and we will print the Medication Guide per FDA to facilitate discussions. The study measures are (1) specific medication information on the leaflet that is hard to comprehend, (2) trigger points for information avoidance and perceived familiarity, and (3) patients' suggestions. An initial coding framework will be developed by two coders to establish themes and subthemes from the transcript data. Refinement of coding will follow, and any disagreement between two coders will be resolved by consulting with a third coder.

\section{Step 2: Eye-Tracking Study}

In the second phase, we will design a web-based mock interface representing medication information in an interactive visualization format. We will use medication information from the FDA's Medication Guides that are provided to patients for designing this mock-up. We will use the open-source software platform Pupil, a wearable, mobile, eye-tracking headset with one scene camera and one infrared-spectrum eye camera for dark pupil detection [28]. The video streams are read using Pupil capture software for real-time pupil detection, gaze mapping, recording, and other functions. We will use a mobile platform to display this information. Patients will be asked to wear the Pupil device and browse the web-based mock-up of medication information on a mobile device. They also will be asked to perform a series of tasks; for example, "find the most common side effect of the drug," "find the storage instructions," "find instructions on whether you can break the pill in half and take it," etc. Patients will be asked to browse the interface data and provide overall feedback in a short semistructured verbal interview that will follow the task segment. The study measures are (1) specific areas in medication information where attention is focused, (2) real-time gaze movement using the Pupil software algorithm, and (3) accuracy, response time, and eye-movement data. For the data analysis, the Gaze History Length option for each surface decides how many recent gaze positions will be used to calculate the heat map based on gaze history. We will use annotation software to label timestamps of specific video gazing to understand the attention locus. Two researchers will assess the transcribed audio interviews to generate trigger points, user-centered design recommendations, and user preferences.

\section{Step 3: Psychological Refractory Period Paradigm Study}

In the third phase, we seek to understand users' variations in their ability to complete two tasks in rapid succession for a goal-directed behavior; in our proposed research design, this understanding will be unpacked by determining the limits of dual-tasking by self-regulation and task-based measurement. The specific methodology for data collection is the PRP task, a tool provided by the National Institutes of Health Science of Behavior Change (SOBC) program [29-31]. The PRP gives specific instructions, which participants must follow as stated on the screen. The time, variation, and accuracy are measured through PRP and results are readily available to be downloaded after the session. The results will help us understand functionalities based on user profile variations, including age, health literacy, or any other physical and mental conditions. Patients will be given the test from the SOBC site from a web browser on a laptop computer. The study measures are (1) response time and (2) task-switching time. For the data analysis, we will note the variations of the response time based on patients' ages and backgrounds to help create design features of buttons and clickable interface functional requirements in the design for the interface.

\section{Study Subjects and Recruitment Methods}

We will recruit 50 patients from our outpatient clinic sites. A research assistant will conduct a telephone interview for cognitive status to ensure potential participants meet the minimum cutoff score of 34 during cognitive screening based on TICS-M test for sound cognitive status and health literacy level [32]. Participants will come to our main site at a lab at the Western University of Health Sciences, Pomona, California. Inclusion criteria will be patients who are aged 18-65 years and have started at least one new medication to develop visualization 
information. The observational study (30 minutes), eye-tracking study (20 minutes), and PRP (10 minutes) will take around one hour, including instructions and filling out consent forms. Patients will be offered a US $\$ 100$ gift card and access to a pharmacist consultation for the duration of the study.

\section{Data Collection}

We propose to collect the following data: (1) observation notes and audio recordings, (2) video data from Pupil on specific locations on gaze mapping, surface detection, and audio streaming data, (3) notes from user observations and audio of the short interviews after the eye-tracking study, and (4) task-switching and task-processing time from PRP.

\section{Sample Size Justifications}

Previous observation and eye-tracking studies used 10-90 participants and 5-40 participants, respectively [17,33,34]. We plan to recruit 50 patients for this aim.

\section{Limitations}

Patients may experience peer pressure to show conformity to medication information. We will have the moderator clarify to patients that the questions have no right or wrong answers. Some errors may result from gaze mapping, surface detection, and algorithm data. Therefore, we will calibrate the software and hardware before each study.

\section{Aim 2: Iteratively Design and Evaluate the AI-Powered, Medication Information Visualization Interface}

\section{Methods Overview}

We will use an AI-based smartphone interface platform for our iterative design of the medication information interface. To develop such a platform, we will use the Amazon SageMaker engine for creating an AI-powered interface with our RNN-based algorithm. This interface will be able to take into account users' interactions and translate the data into a data analytics-based back-end server. This prototype will be developed in Java and Python languages for both android and iOS platforms.

\section{Human Factor-Based Iterative Design}

We will use a human factor-based methodology to iteratively design the app from the functional requirements from Aim 1 using user-centered design principles [12,23,34]. We will use the usability inquiry approach for the iterative design to understand the users' likes, dislikes, and needs [35,36]. The multidisciplinary research team, including 20 clinicians with diverse clinical backgrounds and 12 researchers, will then iteratively review and revise the app based on the written and verbal feedback related to the usability (ie, think-aloud methods), efficiency, and ease of use for 4 months or until no further revisions are identified. App usability will not be measured remotely. A research assistant will recruit clinicians and other team members for the iterative design process related to the front end of the prototype app. We will ask users to interact with the mock-up in the mobile interface and provide them with tasks using think-aloud methods. Think-aloud methods will provide rich verbal data about specific changes and functionalities of the initial mock-up [35,37]. We will audio record and screen record sessions, using Camtasia (TechSmith Corporation), to analyze verbal feedback from the think-aloud method and measure the mouse movements. We will analyze the data from the think-aloud sessions and screen recordings to identify design functionalities, and we will change the design based on feedback. The think-aloud sessions and screen recordings will provide us with specific user comments and interface design preferences. Our design team will incorporate those changes and take users' feedback in an iterative process unless a final design agreement has been reached.

\section{Contextual Medication Information for the Visualization}

Two medication information expert pharmacists will develop contextual medication information-10 pieces of crucial information-that the patients should know [38]. This information may include indications for use, essential warnings, contraindications, storage information, typical side effects, and directions for use. We will use this information in the design of the visualization as well as use just the text version of the information as an intervention for Aim 3.

\section{Usability Testing of the App}

Two usability surveys will be emailed to users. The first survey instrument, which has been validated and created from the research on smartphone apps from the industry, is specifically designed for mobile interface design $[39,40]$. This Likert scale-based survey is based on a heuristic evaluation for easy, quick, and reliable assessment of the mobile user interface design [39]. The second survey instrument is the hierarchical task measure developed by the SOBC program. Hierarchical task analysis helps us to understand how users categorize tasks in hierarchical steps in their minds [41]. The hierarchical reinforcement learning task measures participants' abilities to discover and use higher-order structures in their environment. Participants are presented with 18 stimuli composed of three dimensions: shape, orientation, and border color. The task requires that participants respond to stimuli by pressing one of three keys in response to each of the stimuli. In a "flat" condition, the keys are randomly associated with the shapes so that the participant must learn each association independently. We will iteratively change the design of the visualization based on the survey results.

\section{Study Subjects and Recruitment Methods}

We will recruit patients (18-65 years of age) at clinic sites during the discharge process with access to, and the ability to use, a smartphone device. A research assistant will conduct the modified Telephone Interview for Cognitive Status (TICS-M) test for cognitive status to ensure potential participants meet the inclusion criteria of a minimum cutoff score of 34 for sound cognitive status and health literacy level [32]. Patients will be given a US \$50 gift card for participation. We will load our medication information tool remotely into the users' app and send out an e-survey for ratings and feedback. The data from the usability surveys will be sent to a HIPPA (Health Insurance Portability and Accountability Act)-protected secured server. 


\section{Data Analysis}

For the hierarchical task, the results will be shown in graphs with the percentage of error of participants' abilities to simplify tasks. We will correlate the means of the graphs with the results from the survey instruments for a personalized design.

\section{Sample Size Justifications}

Previous studies have included usability surveys with 96-404 participants [40,41]. We plan to recruit 300 patients from our outpatient clinics at the Western University of Health Sciences.

\section{Limitations}

We may receive numerous users' input about the interface. The research team will apply only the changes that correspond to the current literature of design conforming to patient safety [42].

\section{Aim 3: Test the Prototype for Better Comprehension and Adherence to Medication Information}

Our hypothesis for this aim is that the AI-powered visualization tool in the app improves medication information comprehension and adherence when compared with written medication information and contextual plain-text information.

\section{Study Design}

The study has a single-blind and sequentially randomized design. The full design of the study will include two arms with 2 (modality: app versus app with text) $\times 2$ (format: an app with visualization versus an app with contextual medication text information) $\times 2$ (context: contextual medication information versus information without context) +1 (control: app) design. The design is depicted in Figure 1. The randomized trial using binary options (yes or no) provides the stratification needed to identify groups that are prone to get benefits from the different presentations of medication information. The results from the sequential randomized design will help us detect statistically significant differences using a larger sample size. The first randomization helps with filtering patients who have better information recall regardless of the app. The second randomization helps with finding the actual effects of the tool when compared with different interventions.

Figure 1. Study design. The participants are randomized first at R: one group uses only the app (A) and another group is given the app with medication information text (AT). At time point 1 (T1), after 4 weeks, both groups are evaluated for information recall (>80\%). Then, after a second randomization $(\mathrm{R})$, the participants are divided into the following groups: app with medication information text (AT), app with contextual medication information text (ACT), and app with visualization (AV). T0: baseline; T2: time point 2 (after 12 weeks).

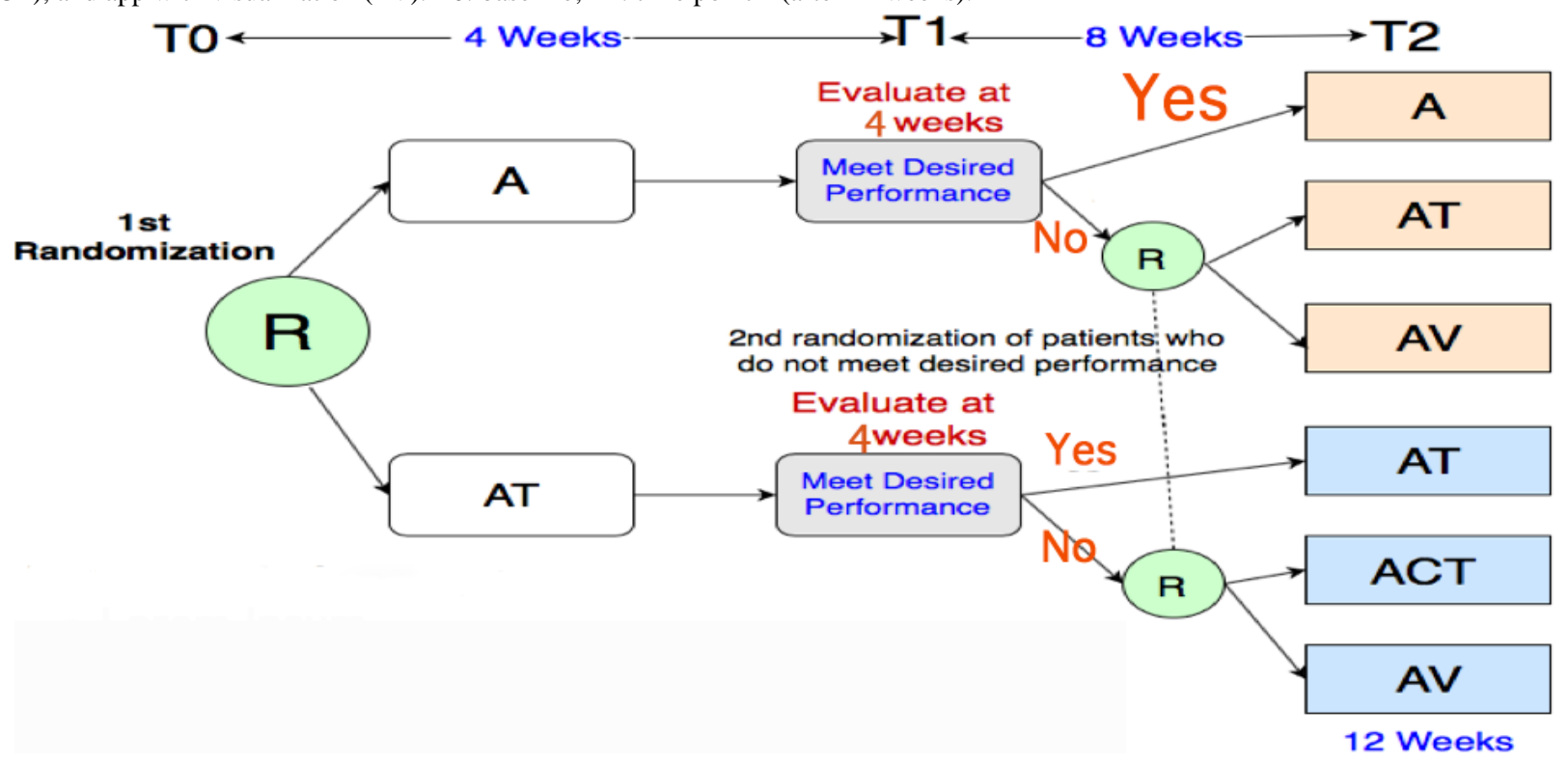

\section{Intervention}

The study will include three intervention conditions loaded into the smartphone app platform. We can better compare the effects of the medication information visualization tool by comparing the outcomes of these three interventions to understand the impacts of our visualization. In the first condition, the app with medication information text (AT), the medication text information will be loaded into the app platform. This medication text is the Medication Guide, which is approved by the FDA. In the second condition, the app with contextual medication information text (ACT) is shown as a printed-text version. This contextual information (ie, 10 pieces of crucial information patients should know) that we included in our visualization will be shown only as the text version in this intervention. In the third condition, an app with visualization
(AV), the interactive app will be loaded with our medication information visualization tool, including the visual format of the contextual information.

\section{Study Subjects and Recruitment Methods}

We will recruit patients (18-65 years of age) from our clinics. Inclusion criteria will include participants who have access to a smartphone, can provide their consent, do not have any cognitive impairment, and who have at least two medications on profile for which we have created a visualization. At the time of recruitment, we will ensure that the patients meet the minimum cutoff score of 34 during cognitive screening using the TICS-M test and health literacy level [32]. Eligible patients will be allocated in a 1:1 ratio to the two arms of the study according to a computer-generated random sequence stratified by third-party software. 


\section{Procedure}

In the first randomization, patients will be randomized into two groups at baseline. The control group (ie, the app-only [A] group) will have the smartphone app with no visualization, and the intervention group will have the app with text information only as in the current Medication Guide (AT) [43]. At 4 weeks, patients from both the control and intervention groups from both arms, who have scores of $80 \%$ or higher in the information recall survey, will continue in the study with the same intervention, in either the A or AT group. Those who do not meet the performance cutoff will be rerandomized further into two groups for each arm. In the second randomization at 4 weeks, the control group from the first arm (A) will have the smartphone app with just text information about medication (AT), and the intervention group will have the smartphone app with the prototype of medication information visualization (AV). The control group from the second arm (AT) will have the app with contextual medication information in a text format (ACT), and the intervention group will have the app with our visualization design (AV). This part of the study will continue for 8 weeks. The monitoring is not a continuous process. Only the principal investigator will have access to the main file and will check the adherence and patient analytics at 4 and 8 weeks.

\section{Survey Tools}

Three survey measures will be used. The first survey will be used to understand information recall; we will design a timed questionnaire, with true or false responses, for each medication in a patient's regimen that includes the 10 pieces of crucial medication information we embedded into the visualization interface. Each patient will be tested on different medications at 4 and 12 weeks to show understanding and knowledge. For example, if a patient takes simvastatin, metformin, and losartan, only those specific medication-related questions will be asked. The second survey will help us to understand the patient's perception of medication knowledge. We will use a validated survey instrument to assess patients' attitudes, confidence, and perceived medication knowledge [44]. This instrument was validated with high internal consistency (Cronbach $\alpha=.833$ ). The third survey will use the 8-item Morisky Medication Adherence Scale (MMAS-8). The MMAS-8, whose scores range from 0 to 8 with lower scores indicating lower adherence, is a widely used tool for self-reported medication adherence; the scale was found to be reliable and significantly associated with adherence control $(P<.05)$, with $93 \%$ sensitivity as well as $53 \%$ specificity for low adherence in a validation study [37].

\section{Data Collection}

We will collect demographics data at baseline. Data will be collected at 4 and 12 weeks. The 12-week time point will be considered the primary endpoint for the study. The AI platform on the smartphone app's back end will provide patient analytics on the usage of the mobile app (ie, intake of medications in the app diary and access frequencies).

\section{Study Measures}

The following study measures will be conducted:
1. Medication information comprehension from the first survey (primary outcome) [45]; the comprehension measure will be a composite of three subscales:

a. Relevance to understanding patients' attitudes,

b. Patients' levels of confidence and knowledge of medication use, measured using a Likert scale, and

c. Information recall subscale, measured using a dichotomous scale (ie, true or false).

2. Survey on patients' perceptions of medication information.

3. Medication adherence (secondary outcome) will be operationalized by the following:

a. MMAS-8 [46] survey score; the dichotomous response categories are yes or no for each item, and

b. Analytics of the patients' daily intake of medications diary from the smartphone app.

\section{Data Analysis}

Demographic information will be presented using descriptive statistics. We will analyze the groups using $t$ tests to detect differences between the means for continuous data. Chi-square analysis will be used to evaluate differences between arms for primary and secondary outcomes. We will use the univariate analysis of variance to calculate the mean differences between groups. If the distribution is not normal, we will use general linear modeling for the data analysis [47,48]. The AI-based analytics data from the smartphone will show us the patients' medication intake and we will correlate that score with our interventions.

\section{Sample Size Justifications}

To detect a 1-point improvement on the Likert scale and $80 \%$ power at the $5 \%$ significance level, the study would require 100 subjects in each group with 1:1 allocation for the second randomization (ie, AT: AV and ACT: AV). To allow for an $11 \%$ loss due to dropouts and those lost to follow-up, we would need to recruit 888 patients, allowing up to $50 \%$ of the patients to move to the second randomization following the first randomization with a score of $80 \%$ or higher in the medication recall questionnaire survey with assigned intervention. Recruitment of 888 patients is also sufficient to detect a 1-point improvement on the MMAS- 8 (the secondary outcome), with $80 \%$ power at $5 \%$ significance. Therefore, our target is to recruit 900 patients.

\section{Limitations}

We expect to enroll a large number of patients for this sequential randomized design. We do not anticipate significant problems because our clinics are excellent sites for recruitment. The recruiting advantage is due to the academic affiliations with faculty and residents. However, given our large sample size requirements, to the extent that we cannot recruit around 888 patients, our study will lack rigor.

\section{Results}

We will conduct preliminary data collection for the studies in 2021. Results are expected to be published in 2022 . 


\section{Discussion}

\section{Overview}

This protocol addresses the problem of creating an AI-powered smartphone app to engage and predict user analytics. This unique idea builds upon an understanding of how users interact with complex medication task information and interactively visualize crucial medication information that frequently is ignored. Previous studies have also successfully shown that informatics platforms have the potential to empower patients [49]. While AI-based algorithms have been used in predicting health outcomes [20,50], very few studies have looked into the user's interaction analytics and ways to build better visualization techniques to engage the user [51-54]. This protocol focuses on creating uniform data standards that are crucial for efficient health information exchange [55]. This protocol provides future researchers and visualization designers with a new and innovative way to design and improve health care smartphone apps.

\section{Conclusions}

This study will lead the future of innovative AI-powered smartphone app design and act as the aid to improve medication risk comprehension, which may ultimately improve medication adherence. The results from this study also open up future research opportunities to understand how patients manage complex medication information and will inform the format and design for innovative AI-powered digital interfaces for Medication Guides.

\section{Acknowledgments}

We give our appreciation to the Western University of Health Sciences for helping and supporting the ideas in this proposal. Also, we acknowledge internal funding that supports this research from the Western University of Health Sciences, College of Pharmacy.

\section{Conflicts of Interest}

None declared.

\section{References}

1. Sage A, Blalock SJ, Carpenter D. Extending FDA guidance to include consumer medication information (CMI) delivery on mobile devices. Res Social Adm Pharm 2017;13(1):209-213. [doi: 10.1016/j.sapharm.2016.01.001] [Medline: 26868207]

2. Cunningham MR. Revolutionizing the patient package insert with infographics. BU Well 2016 May 13;1:5-6.

3. Wolf MS, Davis TC, Shrank WH, Neuberger M, Parker RM. A critical review of FDA-approved Medication Guides. Patient Educ Couns 2006 Sep;62(3):316-322. [doi: 10.1016/j.pec.2006.06.010] [Medline: 16884888]

4. Wolf MS, King J, Wilson EAH, Curtis LM, Bailey SC, Duhig J, et al. Usability of FDA-approved Medication Guides. J Gen Intern Med 2012 Dec;27(12):1714-1720 [FREE Full text] [doi: 10.1007/s11606-012-2068-7] [Medline: 22566170]

5. Hoy MG, Levenshus AB. A mixed-methods approach to assessing actual risk readership on branded drug websites. J Risk Res 2016 Aug 27;21(5):521-538. [doi: 10.1080/13669877.2016.1223160]

6. Chakraborty S, Bouder F. The future of risk communication and the role of the pharmaceutical industry. Curr Drug Saf 2013 Mar;8(1):4-10. [doi: 10.2174/1574886311308010002] [Medline: 23656442]

7. Hayes TL, Larimer N, Adami A, Kaye JA. Medication adherence in healthy elders: Small cognitive changes make a big difference. J Aging Health 2009 Jun;21(4):567-580. [doi: 10.1177/0898264309332836] [Medline: 19339680]

8. Pignone M, DeWalt DA, Sheridan S, Berkman N, Lohr KN. Interventions to improve health outcomes for patients with low literacy. A systematic review. J Gen Intern Med 2005 Mar;20(2):185-192. [doi: 10.1111/j.1525-1497.2005.40208.x] [Medline: 15836553 ]

9. Stilley CS, Bender CM, Dunbar-Jacob J, Sereika S, Ryan CM. The impact of cognitive function on medication management: Three studies. Health Psychol 2010 Jan;29(1):50-55 [FREE Full text] [doi: 10.1037/a0016940] [Medline: 20063935]

10. Hemminger BM, Long T, Saelim B. Comparison of Visualization Techniques for Displaying Medication History to Older Adults. UNC SILS Technical Report. Chapel Hill, NC: University of North Carolina School of Information and Library Science (UNC SILS); 2006 Apr. URL: https://ils.unc.edu/bmh/pubs/Personal Health Records 2006.pdf [accessed 2020-10-30]

11. Wroe AL. Intentional and unintentional nonadherence: A study of decision making. J Behav Med 2002 Aug;25(4):355-372. [doi: 10.1023/a:1015866415552] [Medline: 12136497]

12. Lindquist LA, Go L, Fleisher J, Jain N, Friesema E, Baker DW. Relationship of health literacy to intentional and unintentional non-adherence of hospital discharge medications. J Gen Intern Med 2012 Mar;27(2):173-178. [doi: 10.1007/s11606-011-1886-3] [Medline: 21971600]

13. Brown MT, Bussell JK. Medication adherence: WHO cares? Mayo Clin Proc 2011 Apr;86(4):304-314 [FREE Full text] [doi: 10.4065/mcp.2010.0575] [Medline: 21389250]

14. Amin S, Soliman M, McIvor A, Cave A, Cabrera C. Understanding patient perspectives on medication adherence in asthma: A targeted review of qualitative studies. Patient Prefer Adherence 2020 Mar;14:541-551. [doi: 10.2147/ppa.s234651] 
15. Serper M, Patzer RE, Curtis LM, Smith SG, O'Conor R, Baker DW, et al. Health literacy, cognitive ability, and functional health status among older adults. Health Serv Res 2014 Aug;49(4):1249-1267 [FREE Full text] [doi: 10.1111/1475-6773.12154] [Medline: 24476068]

16. Gupta V, Shivaprakash G, Bhattacherjee D, Udupa K, Poojar B, Sori R, et al. Association of health literacy and cognition levels with severity of adverse drug reactions in cancer patients: A South Asian experience. Int J Clin Pharm 2020 Aug;42(4):1168-1174. [doi: 10.1007/s11096-020-01062-9] [Medline: 32472326]

17. Islam R, Weir C, Del Fiol G. Heuristics in managing complex clinical decision tasks in experts' decision making. IEEE Int Conf Healthc Inform 2014 Sep;2014:186-193 [FREE Full text] [doi: 10.1109/ICHI.2014.32] [Medline: 27275019]

18. Islam R, Mayer J, Clutter J. Supporting novice clinicians cognitive strategies: System design perspective. IEEE EMBS Int Conf Biomed Health Inform 2016 Mar;2016:509-512 [FREE Full text] [doi: 10.1109/BHI.2016.7455946] [Medline: 27275020]

19. Islam R, Weir CR, Jones M, Del Fiol G, Samore MH. Understanding complex clinical reasoning in infectious diseases for improving clinical decision support design. BMC Med Inform Decis Mak 2015 Nov 30;15:101 [FREE Full text] [doi: 10.1186/s12911-015-0221-z] [Medline: 26620881]

20. Fogel AL, Kvedar JC. Artificial intelligence powers digital medicine. NPJ Digit Med 2018;1:5 [RREE Full text] [doi: 10.1038/s41746-017-0012-2] [Medline: 31304291]

21. Labovitz DL, Shafner L, Reyes Gil M, Virmani D, Hanina A. Using artificial intelligence to reduce the risk of nonadherence in patients on anticoagulation therapy. Stroke 2017 May;48(5):1416-1419 [FREE Full text] [doi: 10.1161/STROKEAHA.116.016281] [Medline: 28386037]

22. Roosan D, Li Y, Law A, Truong H, Karim M, Chok J, et al. Improving medication information presentation through interactive visualization in mobile apps: Human factors design. JMIR Mhealth Uhealth 2019 Nov 25;7(11):e15940 [FREE Full text] [doi: 10.2196/15940] [Medline: 31763991]

23. Baysari MT, Westbrook JI. Mobile applications for patient-centered care coordination: A review of human factors methods applied to their design, development, and evaluation. Yearb Med Inform 2018 Mar 10;24(01):47-54. [doi: $10.15265 /$ iy-2015-011]

24. Schnall R, Rojas M, Bakken S, Brown W, Carballo-Dieguez A, Carry M, et al. A user-centered model for designing consumer mobile health (mHealth) applications (apps). J Biomed Inform 2016 Apr;60:243-251 [FREE Full text] [doi: 10.1016/j.jbi.2016.02.002] [Medline: 26903153]

25. Roosan D, Samore M, Jones M, Livnat Y, Clutter J. Big-data based decision-support systems to improve clinicians' cognition. IEEE Int Conf Healthc Inform 2016;2016:285-288 [FREE Full text] [doi: 10.1109/ICHI.2016.39] [Medline: 27990498]

26. Litwin AH, Shafner L, Norton B, Akiyama MJ, Agyemang L, Guzman M, et al. Artificial intelligence platform demonstrates high adherence in patients receiving fixed-dose ledipasvir and sofosbuvir: A pilot study. Open Forum Infect Dis 2020 Aug;7(8):ofaa290 [FREE Full text] [doi: 10.1093/ofid/ofaa290] [Medline: $\underline{\text { 32818140] }}$

27. McLafferty I. Focus group interviews as a data collecting strategy. J Adv Nurs 2004 Oct;48(2):187-194. [doi: 10.1111/j.1365-2648.2004.03186.x] [Medline: 15369499$]$

28. Moritz K, Patera W, Bulling A. Pupil: An open source platform for pervasive eye tracking and mobile gaze-based interaction. arXiv. 2014 Apr. URL: https://arxiv.org/pdf/1405.0006.pdf [accessed 2020-09-27]

29. Tombu M, Jolicœur P. A central capacity sharing model of dual-task performance. J Exp Psychol 2003;29(1):3-18. [doi: 10.1037/0096-1523.29.1.3]

30. The Experiment Factory. URL: http://expfactory.org/experiments/psychological_refractory_period_two_choices/preview [accessed 2020-09-14]

31. Psychological refractory period paradigm task. Science Of Behavior Change. URL: https://scienceofbehaviorchange.org/ measures/psychological-refractory-period-paradigm-task/ [accessed 2020-09-14]

32. Cook SE, Marsiske M, McCoy KJM. The use of the Modified Telephone Interview for Cognitive Status (TICS-M) in the detection of amnestic mild cognitive impairment. J Geriatr Psychiatry Neurol 2009 Jun;22(2):103-109 [FREE Full text] [doi: 10.1177/0891988708328214] [Medline: 19417219]

33. Roosan D, Weir C, Samore M, Jones M, Rahman M, Stoddard GJ, et al. Identifying complexity in infectious diseases inpatient settings: An observation study. J Biomed Inform 2017 Jul;71S:S13-S21 [FREE Full text] [doi: 10.1016/j.jbi.2016.10.018] [Medline: 27818310]

34. Duque A, Vázquez C. Double attention bias for positive and negative emotional faces in clinical depression: Evidence from an eye-tracking study. J Behav Ther Exp Psychiatry 2015 Mar;46:107-114. [doi: 10.1016/j.jbtep.2014.09.005] [Medline: 25305417]

35. Srinivas P, Cornet V, Holden R. Human factors analysis, design, and evaluation of Engage, a consumer health IT application for geriatric heart failure self-care. Int J Hum Comput Interact 2017;33(4):298-312 [FREE Full text] [doi: 10.1080/10447318.2016.1265784] [Medline: 30429638]

36. Ahmed I, Ahmad NS, Ali S, Ali S, George A, Saleem Danish H, et al. Medication adherence apps: Review and content analysis. JMIR Mhealth Uhealth 2018 Mar 16;6(3):e62 [FREE Full text] [doi: 10.2196/mhealth.6432] [Medline: 29549075] 
37. Morisky DE, Ang A, Krousel-Wood M, Ward HJ. Predictive validity of a medication adherence measure in an outpatient setting. J Clin Hypertens (Greenwich) 2008 May;10(5):348-354. [doi: 10.1111/j.1751-7176.2008.07572.x] [Medline: $\underline{18453793}$

38. Bann CM, McCormack LA, Berkman ND, Squiers LB. The Health Literacy Skills Instrument: A 10-item short form. J Health Commun 2012;17 Suppl 3:191-202. [doi: 10.1080/10810730.2012.718042] [Medline: 23030570]

39. Islam R, Weir C, Del Fiol G. Clinical complexity in medicine: A measurement model of task and patient complexity. Methods Inf Med 2016;55(1):14-22 [FREE Full text] [doi: 10.3414/ME15-01-0031] [Medline: 26404626]

40. Ali A, Alrasheedi M, Ouda A, Capretz LF. A study of the interface usability issues of mobile learning applications for smart phones from the user's perspective. Int J Integr Technol Educ 2014 Dec 31;3(4):1-16. [doi: 10.5121/ijite.2014.3401]

41. Hoehle H, Venkatesh V. Mobile application usability: Conceptualization and instrument development. MIS Q 2015 Feb 2;39(2):435-472. [doi: 10.25300/misq/2015/39.2.08]

42. Carayon P, Wetterneck TB, Rivera-Rodriguez AJ, Hundt AS, Hoonakker P, Holden R, et al. Human factors systems approach to healthcare quality and patient safety. Appl Ergon 2014 Jan;45(1):14-25 [FREE Full text] [doi: 10.1016/j.apergo.2013.04.023] [Medline: 23845724]

43. Medication guides for certain prescription products. US Food and Drug Administration. URL: http://www.fda.gov/consumers/ consumer-updates/medication-guides-certain-prescription-products [accessed 2020-06-27]

44. Okere AN, Renier CM, Morse J. Development and validation of a survey to assess patient-perceived medication knowledge and confidence in medication use. J Nurs Meas 2014;22(1):120-134. [doi: 10.1891/1061-3749.22.1.120] [Medline: 24851668]

45. Aikin KJ, O’Donoghue AC, Swasy JL, Sullivan HW. Randomized trial of risk information formats in direct-to-consumer prescription drug advertisements. Med Decis Making 2011 Jun 20;31(6):E23-E33. [doi: 10.1177/0272989x11413289]

46. Morisky DE, Green LW, Levine DM. Concurrent and predictive validity of a self-reported measure of medication adherence. Med Care 1986 Jan;24(1):67-74. [doi: 10.1097/00005650-198601000-00007] [Medline: 3945130]

47. Pham JA, Pierce W, Muhlbaier L. A randomized, controlled study of an educational intervention to improve recall of auxiliary medication labeling and adherence to antibiotics. SAGE Open Med 2013;1:1-8. [doi: 10.1177/2050312113490420] [Medline: 26770673]

48. Wolf MS, Davis TC, Curtis LM, Bailey SC, Knox JP, Bergeron A, et al. A patient-centered prescription drug label to promote appropriate medication use and adherence. J Gen Intern Med 2016 Dec;31(12):1482-1489 [FREE Full text] [doi: 10.1007/s11606-016-3816-x] [Medline: 27542666]

49. Roosan D, Hwang A, Roosan MR. Pharmacogenomics cascade testing (PhaCT): A novel approach for preemptive pharmacogenomics testing to optimize medication therapy. Pharmacogenomics J 2020 Aug 25:1-7. [doi: 10.1038/s41397-020-00182-9] [Medline: 32843688]

50. Islam F, Shoilee SBA, Shams M, Rahman RM. Potential risk factor analysis and risk prediction system for stroke using fuzzy logic. In: Proceedings of the Computer Science On-line Conference: Artificial Intelligence Trends in Intelligent Systems. 2017 Apr Presented at: Computer Science On-line Conference: Artificial Intelligence Trends in Intelligent Systems; April 26-29, 2017; Prague, Czech Republic p. 262-272. [doi: 10.1007/978-3-319-57261-1 26]

51. Roosan D, Law AV, Karim M, Roosan M. Improving team-based decision making using data analytics and informatics: Protocol for a collaborative decision support design. JMIR Res Protoc 2019 Dec 27;8(11):e16047 [FREE Full text] [doi: 10.2196/16047] [Medline: 31774412]

52. Roosan R, Karim M, Chok J, Roosan M. Operationalizing healthcare big data in the electronic health records using a heatmap visualization technique. In: Proceedings of the 13th International Joint Conference on Biomedical Engineering Systems and Technologies. 2020 Presented at: 13th International Joint Conference on Biomedical Engineering Systems and Technologies; February 24-26, 2020; Valletta, Malta p. 361-368. [doi: 10.5220/0008912503610368]

53. Saket B, Endert A, Stasko J. Beyond usability and performance: A review of user experience-focused evaluations in visualization. In: Proceedings of the Sixth Workshop on Beyond Time and Errors on Novel Evaluation Methods for Visualization. 2016 Oct Presented at: Sixth Workshop on Beyond Time and Errors on Novel Evaluation Methods for Visualization; October 24, 2016; Baltimore, MD p. 133-142. [doi: 10.1145/2993901.2993903]

54. Roosan D, Del Fiol G, Butler J, Livnat Y, Mayer J, Samore M, et al. Feasibility of population health analytics and data visualization for decision support in the infectious diseases domain. Appl Clin Inform 2017 Dec 16;07(02):604-623. [doi: 10.4338/aci-2015-12-ra-0182]

55. Roosan D, Hwang A, Law AV, Chok J, Roosan MR. The inclusion of health data standards in the implementation of pharmacogenomics systems: A scoping review. Future Med 2020 Oct 30:2020-0066 [FREE Full text] [doi: 10.2217/pgs-2020-0066]

\section{Abbreviations}

A: app only

ACT: app with contextual medication information text

AI: artificial intelligence

ANN: artificial neural network 
AT: app with medication information text

AV: app with visualization

FDA: US Food and Drug Administration

HIPPA: Health Insurance Portability and Accountability Act

MMAS-8: 8-item Morisky Medication Adherence Scale

PRP: psychological refractory period

RNN: recursive neural network

SOBC: Science of Behavior Change

TICS-M: modified Telephone Interview for Cognitive Status

Edited by G Eysenbach; submitted 20.06.20; peer-reviewed by R Holden, $R$ Mickelson; comments to author 25.08.20; revised version received 08.10.20; accepted 13.10.20; published 09.11.20

Please cite as:

Roosan D, Chok J, Karim M, Law AV, Baskys A, Hwang A, Roosan MR

Artificial Intelligence-Powered Smartphone App to Facilitate Medication Adherence: Protocol for a Human Factors Design Study

JMIR Res Protoc 2020;9(11):e21659

URL: http://www.researchprotocols.org/2020/11/e21659/

doi: $10.2196 / 21659$

PMID: 33164898

CDon Roosan, Jay Chok, Mazharul Karim, Anandi V Law, Andrius Baskys, Angela Hwang, Moom R Roosan. Originally published in JMIR Research Protocols (http://www.researchprotocols.org), 09.11.2020. This is an open-access article distributed under the terms of the Creative Commons Attribution License (https://creativecommons.org/licenses/by/4.0/), which permits unrestricted use, distribution, and reproduction in any medium, provided the original work, first published in JMIR Research Protocols, is properly cited. The complete bibliographic information, a link to the original publication on http://www.researchprotocols.org, as well as this copyright and license information must be included. 